\title{
c-MET immunostaining in colorectal carcinoma is associated with local disease recurrence
}

\author{
Jaudah Al-Maghrabi ${ }^{1,2,3^{*}}$, Eman Emam ${ }^{2,4}$, Wafaey Gomaa ${ }^{2,5}$, Moaath Saggaf ${ }^{2}$, Abdelbaset Buhmeida ${ }^{3}$, \\ Mohammad Al-Qahtani ${ }^{3}$ and Mahmoud Al-Ahwal ${ }^{1,6}$
}

\begin{abstract}
Background: Increased mesenchymal-epithelial transition factor gene (c-MET) expression in several human malignancies is related to increased tumour progression. The aim of the present study is to explore the relationship between immunohistochemical expression of c-MET in colorectal carcinoma (CRC) and the clinicopathological characteristics and follow up data, to compare the expression of c-MET in primary CRC and its metastasis in lymph nodes and to test its validity as independent prognostic factor.

Methods: Hundred and thirty-five archival CRC and nodal metastases samples were collected from King Abdulaziz University Hospital, Saudi Arabia. Tissue microarrays were constructed and immunohistochemistry was done to detected c-MET protein expression. Appropriate statistical analysis was performed.

Results: High c-MET immunostaining was significantly associated with tumour size larger than $5 \mathrm{~cm}(p<0.003)$ and in left colon subsite $(p<0.05)$. There was no significant correlation between $c-M E T$ protein expression and age, sex, degree of differentiation, tumour invasion, presence of nodal metastasis, lymphovascular invasion, status of surgical resection margin, or presence of distant metastasis. Furthermore, no association between c-MET protein expression and disease free survival. High protein expression of c-MET is associated with the incidence of local disease recurrence $(p<0.012)$.

Conclusion: C-MET is a new promising target that may help in understanding the pathogenesis of CRC, and to be used as independent prognostic biomarker to predict local disease recurrence in CRC. Further molecular in vitro and in vivo studies are required to pursue c-MET as potential molecular marker of metastases and test the possibility of its incorporation as a new targeted therapeutic target.
\end{abstract}

Keywords: Colorectal carcinoma, c-met proto-oncogene, Clinicopathological characteristics, Prognosis

\section{Background}

Colorectal carcinoma (CRC) is among the most common neoplasms affecting the industrialised nations. According to the Saudi Arabian National Cancer Registry, CRC is accounting for $11.3 \%$ of all newly diagnosed cases in year 2009. This cancer ranked first among male population and third among female population [1]. The prognosis of colorectal carcinoma is controlled by variable factors which are

\footnotetext{
* Correspondence: jalmaghrabi@hotmail.com

${ }^{1}$ Scientific Chair for Colorectal Cancer, King Abdulaziz University, Jeddah, Saudi Arabia

${ }^{2}$ Department of Pathology, Faculty of Medicine, King Abdulaziz University, P.O. Box 80205, Jeddah 21589, Saudi Arabia

Full list of author information is available at the end of the article
}

classified by the College of American Pathologists [2] into; biological and clinical factors as tumour stage, grade, and molecular factors as genetic markers, DNA contents and markers of angiogenesis and proliferation. According to UICC-TNM and American Joint Committee on Cancer classifications [3] tumour extent, lymph node status, tumour grade, and the assessment of lymphatic and venous invasion are still the most relevant clinicopathological prognostic factors. However, tumours of the same stage can follow significantly different clinical courses, indicating a necessity for the identification of novel prognostic factors, including molecular markers. Therefore, an active search is going on to find powerful new prognostic and 
predictive molecular markers for identifying high-risk patients who would benefit from individually tailored treatment options [4-6].

One of the most promising biological markers in the pathogenesis of CRC is the proto-oncogenes c-MET (mesenchymal-epithelial transition factor gene) which encodes the tyrosine kinase receptor for hepatocyte growth factor/ scatter factor (HGF/SF). Interaction between HGF and cMET plays a key role in cellular proliferation, survival, migration, and invasion. The HGF/c-MET axis contributes a critical physiological function in embryogenesis, angiogenesis, and wound healing $[7,8]$. Under normal conditions c-MET is expressed in vascular cells, lymphatic endothelial cells and hematopoietic cells. Somatic mutation and overexpression of c-MET gene has been described in variety of solid organ tumours including carcinoma of lung, bladder, kidney, thyroid and CRC [7, 9-11]. Over expression of c-MET in such tumours is claimed to be related to increased tumour cell motility, invasiveness, and angiogenesis which may stimulate tumour metastatic ability and contribute to tumour aggressiveness [12-14].

The aim of the present study is to explore the relationship between immunostaining of c-MET in CRC and the clinicopathological characteristics and follow up data, to compare c-MET immunostaining in primary CRC and its metastasis in lymph nodes, and to test its validity as independent prognostic factor.

\section{Methods}

\section{Patients}

The current study involved 135 paraffin blocks retrieved from patient's materials in the archives of the Pathology Department, King Abdulaziz University, Jeddah, Saudi Arabia covering the period from January 1995 to December 2010. Paraffin blocks were sliced and stained routinely with haematoxylin and eosin to evaluate histopathological characteristics of the tumours as well as for histological grading and staging. Clinical parameters of patients were collected from the patient's medical records and listed in Table 1. The study was approved by the Research Committee of the Biomedical Ethics Unit, Faculty of Medicine, King Abdulaziz University, Jeddah, Saudi Arabia. All patients included in this study gave an informed written consent for utilisation of their material in research and was accepted by Research Committee of the Biomedical Ethics Unit.

\section{Tissue microarray construction}

A tissue microarray (TMA) was constructed from 135 primary CRC and 49 corresponding lymph nodes showing tumour metastasis. Two cylindrical cores of $1.5 \mathrm{~mm}$ in diameter were selected from donor paraffin blocks and arrayed in 6 recipient paraffin blocks using the automated tissue arrayer (MASTER 3D HISTECH). Normal placenta tissue was used as control tissue to help orientation of
Table 1 Clinicopathological parameters of cases $(n=135)$

\begin{tabular}{|c|c|c|}
\hline \multicolumn{2}{|l|}{ Parameter } & \multirow{2}{*}{$\frac{\text { Number (\%) }}{67 \text { (49.6\%) }}$} \\
\hline Sex & Male & \\
\hline & Female & 68 (50.4 \%) \\
\hline \multirow[t]{3}{*}{ Grade } & Well-differentiated & $31(23 \%)$ \\
\hline & Moderately-differentiated & $85(63 \%)$ \\
\hline & Poorly-differentiated & $19(14 \%)$ \\
\hline \multirow[t]{2}{*}{ Age } & $<60$ years & $72(53.3 \%)$ \\
\hline & $\geq 60$ years & $63(46.7 \%)$ \\
\hline \multirow[t]{3}{*}{ Tumour location } & Right colon & $38(28.1 \%)$ \\
\hline & Left colon & $83(61.5 \%)$ \\
\hline & Rectum & $14(10.4 \%)$ \\
\hline \multirow[t]{2}{*}{ Tumour size } & $<5 \mathrm{~cm}$ & $56(\% 41.5)$ \\
\hline & $\geq 5 \mathrm{~cm}$ & 79 (58.5\%) \\
\hline \multirow[t]{4}{*}{ Primary tumour } & $\mathrm{T} 1$ & $3(2.2 \%)$ \\
\hline & $\mathrm{T} 2$ & $14(10.4 \%)$ \\
\hline & T3 & $108(80 \%)$ \\
\hline & $\mathrm{T} 4$ & $10(7.4 \%)$ \\
\hline \multirow[t]{3}{*}{ Nodal metastasis } & Positive & $63(46.7 \%)$ \\
\hline & Negative & $68(50.3 \%)$ \\
\hline & Cannot be assessed & $4(3 \%)$ \\
\hline \multirow[t]{2}{*}{ Distant metastasis } & Positive & $40(29.6 \%)$ \\
\hline & Negative & $95(70.4 \%)$ \\
\hline \multirow[t]{2}{*}{ Lymphovascular invasion } & Positive & $20(14.8 \%)$ \\
\hline & Negative & $115(85.2 \%)$ \\
\hline \multirow[t]{2}{*}{ Margin status } & Involved & $9(6.7 \%)$ \\
\hline & Free & $126(93.3 \%)$ \\
\hline \multirow[t]{2}{*}{ Local disease recurrence } & Recurrence & $48(35.6 \%)$ \\
\hline & No recurrence & $87(64.4 \%)$ \\
\hline \multirow[t]{3}{*}{ Survival } & Died of disease & $26(19.3 \%)$ \\
\hline & Alive & 89 (65.9\%) \\
\hline & Not available & 20 (14.8\%) \\
\hline
\end{tabular}

T1: Tumour invades submucosa

T2: Tumour invades muscularis propria

T3: Tumour invades through the muscularis propria into the subserosa or into non-peritonealised pericolic or perirectal tissues

T4: Tumour directly invades other organs or structures, and/or perforates visceral peritoneum

samples in each TMA block. TMA blocks were sliced into 4-micron meter sections embedded in sialinated slides in order to be stained by immunohistochemical staining.

\section{Immunohistochemistry of tissue microarray}

Immunohistochemical staining of CRC samples were using antibody to protein c-MET was performed by avidin biotin procedure following manufacturer's kit instructions. The antibody used was a monoclonal mouse Anti-Human c-MET (Dako Cytomation Norden A/S, Glostrup, Denmark, dilution 1:100). IHC procedure was 
carried out using an automatic immunostainer (Ventana Bench Mark XT, Ventana Inc., Tucson, AZ). In each analysis, positive controls were used consisting of CRC samples previously shown to stain with this antibody. Tris-buffered saline in place of the primary antibody was used as a negative control.

\section{Interpretation of immunohistochemical staining}

c-MET protein positivity appeared as yellow to brown staining in tumour cell cytoplasm and/or cell membrane. The immune reaction was evaluated semiquantitavely according to staining intensity taking into account the percentage of cells staining at a given intensity, following the scoring system given by Takeuchi et al. [15] on a scale from 0 to 3 , with grade 0 : negative, 1 : weak, 2 : moderate, and 3: intense. When dichotomised for statistical risk assessment, negative $(-)$ and weak $(+)$ staining were defined as low immunostaining, while moderate $(++)$ and intense $(+++)$ staining were included in high immunostaining category.

\section{Statistical analysis}

Differences between two groups of patients on one variable were tested by using Mann Whitney test while between three groups of patients by Kruskal Wallis test was used. Non-parametric chi-square was used to test variance along one variable. Binary logistic regression analysis was used to predict lymph noel metastasis, distant metastasis, surgical resection margins involvement, lymphovascular invasion, and local disease recurrence in relation immunoexpression of c-MET. Estimated odds ratio \{exponential (B)\}, $95 \%$ confidence interval (CI) for $\exp (\mathrm{B})$. The Kaplan-Meier procedure was used to calculate the disease-free survival probabilities and the Log Rank test was used to compare the difference between survivals. Time was calculated from the date of diagnosis to the appearance of disease relapse (or date last seen disease-free). Statistical procedures were performed using SPSS $^{\circledR}$ Release 16.0. Statistical significance was determined at $p$ value of $\leq 0.05$ and was 2 -sided.

\section{Results}

\section{c-MET immunostaining profiles}

In CRC, c-MET protein expression was observed as in tumour cells, but was negative in inflammatory cells, endothelial cells, smooth muscle cells or fibroblasts. c-MET immunostaining positivity was observed as yellow-brown staining observed in the cytoplasm and cell membrane. (Fig. 1) Cases with low c-MET protein expression outnumbered cases showing high c-MET protein expression; low expression: 87 cases (64.4\%) and high expression: 48 cases (35.6\%). There was no difference between $\mathrm{c}$-MET protein expression in primary tumour and in lymph node metastasis (Table 2).

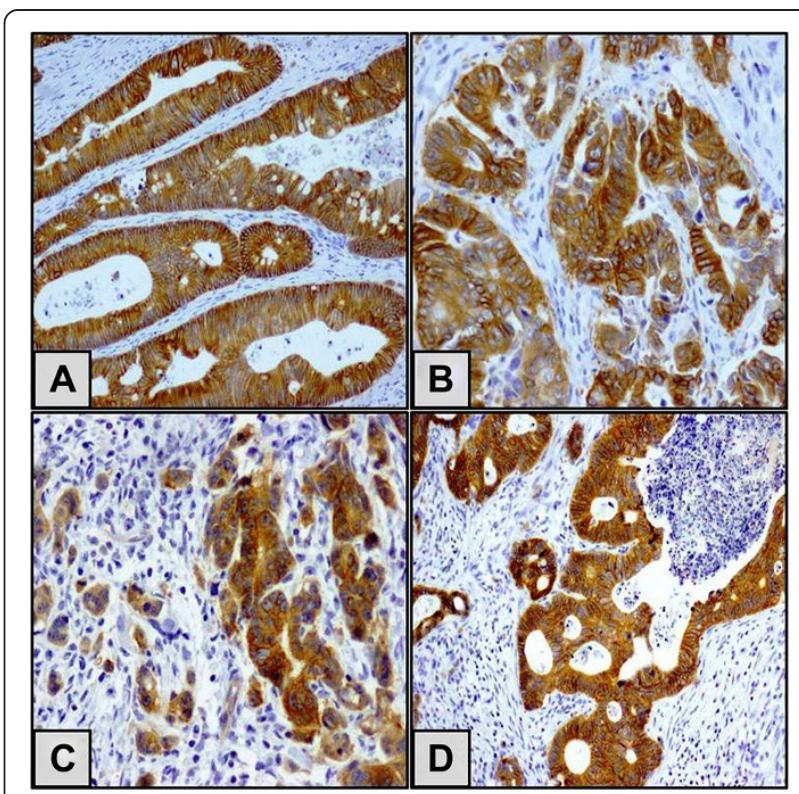

Fig. 1 Immunohistochemical labelling of c-MET in CRC and nodal metastasis using anti c-MET antibody. Diaminobenzidine was used as chromogen and haematoxylin as counterstain. A combined cytoplasmic and membranous immunostaining is shown in well differentiated CRC (a), moderately differentiated CRC (b), poorly differentiated CRC (c) and in lymph node metastasis (d). Original magnification used is $200 \mathrm{x}$

The relationship between c-MET immunostaining and clinicopathological features of CRCs

There was no significant correlation between c-MET expression and age, sex, degree of differentiation, depth of tumour invasion, tumour stage, presence of nodal metastasis, lymphovascular invasion, status of surgical margins and presence of nodal distant metastasis. On the other hand, high c-MET immunostaining was significantly associated with tumour sizes larger than $5 \mathrm{~cm}$ and in left colon location (Table 3).

\section{The relationship between c-MET protein expression and survival outcome}

The survival curves for high- and low c-MET groups were assessed by the Kaplan-Meier method and compared by a log-rank test which showed no association of c-MET protein expression with disease-free survival (Log rank $=1.142$, and $p=0.285$ ) (Fig. 2). Regression

Table 2 Categories of c-MET protein immunoexpression

\begin{tabular}{llll}
\hline & $\begin{array}{l}\text { Primary tumour } \\
(n=135)\end{array}$ & $\begin{array}{l}\text { Nodal Metastasis } \\
(n=61)\end{array}$ & $p$ value \\
\hline Low expression & $87 / 135(64.4 \%)$ & $46 / 61(75.4 \%)$ & $0.129^{* *}$ \\
High expression & $48 / 135(35.6 \%)$ & $15 / 61(24.6 \%)$ & \\
$p$ value & $<0.001^{*}$ & $<0.001^{*}$ & \\
\hline
\end{tabular}

"One sample non-parametric chi-square test

${ }^{* *}$ Mann-Whitney test 
Table 3 Association of c-MET protein immunoexpression with clinicopathological parameters

\begin{tabular}{ll}
\hline Parameter & $p$ value \\
\hline Grade & $0.079^{*}$ \\
Sex & $0.768^{* *}$ \\
Age & $0.566^{* *}$ \\
Tumour location & $0.05^{*}$ \\
Tumour size & $0.003^{* * *}$ \\
Depth of invasion [2] & $0.092^{*}$ \\
Nodal metastasis & $0.682^{* *}$ \\
Lymphovascular invasion & $0.287^{* *}$ \\
Margin status & $0.886^{* *}$ \\
Local disease recurrence & $0.012^{* * *}$ \\
Distant metastasis & $0.206^{* *}$
\end{tabular}

"Kruskal-Wallis Test

${ }^{* *}$ Mann-Whitney test

${ }^{* * *}$ Signficantly associated with c-MET overexpression

analysis for c-MET immunoexpression revealed that high protein expression is associated with the incidence of recurrence and could be an independent predictor of occurrence of recurrence (Table 4).

\section{Discussion}

CRC remains the second leading cause of cancer-related death worldwide despite recent advances in adjuvant chemotherapeutic regimens [16]. Recent researches are concerned about better understanding of the pathogenesis

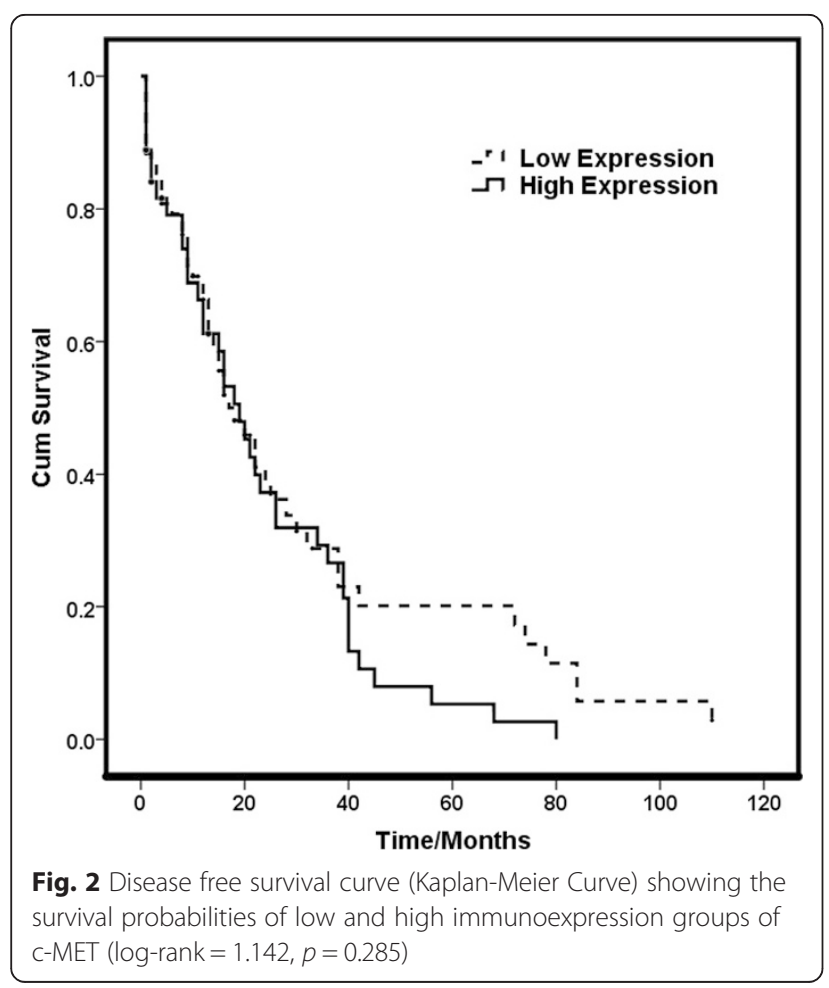

and biological characteristics of CRC aiming at development of new targets for therapy. The role of c-MET signalling has not been fully elucidated in CRC. c-MET is aberrantly activated in many human cancers through diverse mechanisms, including point mutations, gene amplification, transcriptional up-regulation, or ligand autocrine loops.

The present study showed that c-MET protein is concomitantly overexpressed in both primary tumours and in nodal metastasis which is similar other studies findings $[17,18]$. The current study demonstrates that high protein expression of c-MET protein using immunohistochemical technique was demonstrated to be significantly associated with large tumour size, and left side location of the tumour. However, other studies Zeng et al., [19] and Liu et al., [20] did not find any correlation between c-MET and tumour site or size $[19,20]$. We did not find any significant correlation between c-MET protein overexpression and tumour stage, distant metastasis, or lymph node positivity contrasting results of previous studies $[15,19,20]$. In Takeuchi et al. study, they found high c-MET immunostaining was associated with advanced stages [15]. Although the same scoring system was used, their sample size was relatively smaller than in the current study. Zeng et al. observed C-MET gene amplification in advanced primary tumour stages. Additionally, Liu et al. showed c-MET mRNA and protein overexpression in primary CRC with high stage and positive nodal metastasis [20]. The discrepancy from the present study may be due to smaller number of tumours. In the current study, there was no association between c-MET protein overexpression and disease free survival which is similar to previous report [18]. In another studies, c-MET immunostaining was found to be associated with overall survival $[17,21]$. This discrepancy may be attributed to small sample size and using a different immunostaining scoring system.

Significant statistical correlation was identified between c-MET protein overexpression and tumour recurrence. This result is comparable to those of Saigusa et al. [9] that investigated the relation between c-MET protein expression and distant recurrence following preoperative chemotherapy in rectal carcinoma and the effect of cMET inhibition on tumour cell growth after radiotherapy. They demonstrated that C-MET protein overexpression is related to tumour recurrence and is associated with worse prognosis and suggested that inhibition of c-MET is a potential new strategy for reduction of distant recurrence of rectal carcinoma after preoperative chemotherapy.

In addition to association of c-MET activation with tumour progression, previous studies demonstrated that the HGF/c-MET signalling pathway plays a role in angiogenesis and lymphangiogenesis by promoting 
Table 4 Regression analysis for c-MET protein immunoexpression

\begin{tabular}{lcll}
\hline Variable & Exp (B) & $95 \%$ Cl for exp (B) & $p$ value \\
\hline Nodal Metastasis & 1.163 & $0.566-2.387$ & 0.681 \\
Distant metastasis & 1.682 & $0.750-3.770$ & 0.207 \\
Surgical resection margins & 1.111 & $0.265-4.657$ & 0.885 \\
Lymphovascular invasion & 1.792 & $0.608-5.278$ & 0.290 \\
Local disease recurrence & 3.322 & $1.264-8.732$ & $0.015^{*}$ \\
\hline
\end{tabular}

${ }^{\circ}$ c-MET overexpression is an independent predictor of the incidence of local disease recurrence

the growth of endothelial cells, increasing the expression of pro-angiogenic mediators, such as vascular endothelial growth factor, and suppressing the activity of thrombospondin 1-a negative regulator of angiogenesis. Considering the relevant role of c-MET in angiogenesis, activated c-MET is considered as adjuvant pro-metastatic gene for many tumour types. Novel therapeutic strategies, which focus on the simultaneous blockade of such proto-oncogene, have been recently proposed to improve the treatment outcome [22-24].

The limitation of the current study includes including missing some follow-up data, short survival time in a number of patients.

\section{Conclusions}

In summary, in the present study the association of high c-MET immunostaining with larger tumours supports involvement of c-MET in tumour progression. On the other hand, c-MET is a promising target that may help in understanding the pathogenesis of $\mathrm{CRC}$, and to be used as independent prognostic biomarker to predict local disease recurrence in CRC. Further molecular in vitro and in vivo studies are required to pursue c-MET as potential molecular marker of metastases and test the possibility of its incorporation as a new targeted therapeutic target.

\section{Abbreviations \\ CRC: Colorectal carcinoma; HGF: Hepatocyte growth factor; UICC: Union for International Cancer Control; TNM: Tumour, Node Metastasis; TMA: Tissue microarray.}

\section{Competing interests}

The authors confirm that no part of this work has been submitted or published elsewhere and that there are no conflicts of interest.

\footnotetext{
Authors' contributions

Jaudah Al-Maghrabi shared in histological examination and selection of paraffin blocks included in study, contributed to the design of the study, and revised the manuscript. Eman Emam shared in histological examination and selection of paraffin blocks included in study, shared in scoring of immunostaining, and revised the manuscript. Wafaey Gomaa designed TMA, shared in paraffin blocks selection, performed statistical analysis, and drafted the manuscript. Moaath Saggaf shared in scoring of immunostaining. Abdelbaset Buhmeida contributed to the design of the study, and revised the manuscript. Mohammad Al-Qahtani contributed to the design of the study, and revised the manuscript. Mahmoud Al-Ahwal reviewed the clinical data and contributed to the design of the study and revised the manuscript. The
}

manuscript has been read and approved by all of the authors, the requirements for authorship have been met, and each author believes that the manuscript represents honest work.

\section{Acknowledgements}

This work was supported by Ministry of Higher Education and King Abdulaziz City for Science and Technology (KACST) grant 11-BIO1524-03 and the Scientific Chair for Colorectal Cancer, King Abdulaziz University, Jeddah, Saudi Arabia.

\section{Author details}

'Scientific Chair for Colorectal Cancer, King Abdulaziz University, Jeddah, Saudi Arabia. ${ }^{2}$ Department of Pathology, Faculty of Medicine, King Abdulaziz University, P.O. Box 80205, Jeddah 21589, Saudi Arabia. ${ }^{3}$ Center of Excellence in Genomic Medicine Research, King Abdulaziz University, Jeddah, Saudi Arabia. ${ }^{4}$ Department of Pathology, Faculty of Medicine, Alexandria University, Alexandria, Egypt. ${ }^{5}$ Department of Pathology, Faculty of Medicine, Minia University, Al Minia, Egypt. ${ }^{6}$ Department of Medicine, Faculty of Medicine, King Abdulaziz University, Jeddah, Saudi Arabia.

Received: 30 December 2014 Accepted: 28 September 2015

Published online: 12 October 2015

\section{References}

1. Cancer Incidence Report of Saudi Arabia [http://www.chs.gov.sa/Ar/ HealthRecords/CancerRegistry/CancerRegistryReports/ Incidence\%20Report\%202009.pdf]

2. Compton CC, Fielding LP, Burgart $L$, Conley B, Cooper HS, Hamilton SR, et al. Prognostic factors in colorectal cancer. College of American Pathologists Consensus Statement. Arch Pathol Lab Med 2000. 1999;124(7):979-94.

3. Puppa G, Sonzogni A, Colombari R, Pelosi G. TNM staging system of colorectal carcinoma: a critical appraisal of challenging issues. Arch Pathol Lab Med. 2010;134(6):837-52.

4. Dallol A, Al-Maghrabi J, Buhmeida A, Gari MA, Chaudhary AG, Schulten HJ, et al. Methylation of the Polycomb Group Target Genes (PCGT) is a Possible Biomarker for Favorable Prognosis in Colorectal Cancer. Cancer Epidemiol Biomarkers Prev. 2012;21(11):2069-75.

5. Al-Maghrabi J, Gomaa W, Buhmeida A, Al-Qahtani M, Al-Ahwal M. Decreased immunoexpression of standard form of CD44 is an independent favourable predictor of nodal metastasis in colorectal carcinoma. Anticancer Res. 2012;32(8):3455-61.

6. Al-Maghrabi J, Buhmeida A, Emam E, Syrjanen K, Sibiany A, Al-Qahtani M, et al. Cyclooxygenase-2 expression as a predictor of outcome in colorectal carcinoma. World J Gastroenterol. 2012;18(15):1793-9.

7. Ma PC, Tretiakova MS, Mackinnon AC, Ramnath N, Johnson C, Dietrich S, et al. Expression and mutational analysis of MET in human solid cancers. Genes, chromosomes \& cancer. 2008;47(12):1025-37.

8. Onozato R, Kosaka T, Kuwano H, Sekido Y, Yatabe Y, Mitsudomi T. Activation of MET by gene amplification or by splice mutations deleting the juxtamembrane domain in primary resected lung cancers. J Thorac Oncol. 2009:4(1):5-11.

9. Saigusa S, Toiyama Y, Tanaka K, Yokoe T, Fujikawa H, Matsushita K, et al. Inhibition of HGF/CMET expression prevents distant recurrence of rectal cancer after preoperative chemoradiotherapy. Int J Oncol. 2012;40(2):583-91.

10. Cecchi F, Rabe DC, Bottaro DP. Targeting the HGF/Met signalling pathway in cancer. Eur J Cancer. 2010;46(7):1260-70.

11. Miyata Y, Sagara Y, Kanda S, Hayashi T, Kanetake H. Phosphorylated hepatocyte growth factor receptor/c-Met is associated with tumor growth and prognosis in patients with bladder cancer: correlation with matrix metalloproteinase-2 and -7 and E-cadherin. Hum Pathol. 2009;40(4):496-504.

12. Kammula US, Kuntz EJ, Francone TD, Zeng Z, Shia J, Landmann RG, et al. Molecular co-expression of the c-Met oncogene and hepatocyte growth factor in primary colon cancer predicts tumor stage and clinical outcome. Cancer Lett. 2007;248(2):219-28.

13. Herynk MH, Zhang J, Parikh NU, Gallick GE. Activation of Src by c-Met overexpression mediates metastatic properties of colorectal carcinoma cells. J Exp Ther Oncol. 2007;6(3):205-17.

14. Bauer TW, Fan F, Liu W, Johnson M, Parikh NU, Parry GC, et al. Insulinlike growth factor-I-mediated migration and invasion of human 
colon carcinoma cells requires activation of c-Met and urokinase plasminogen activator receptor. Ann Surg. 2005;241(5):748-56. discussion 756-748

15. Takeuchi H, Bilchik A, Saha S, Turner R, Wiese D, Tanaka M, et al. c-MET expression level in primary colon cancer: a predictor of tumor invasion and lymph node metastases. Clin Cancer Res. 2003;9(4):1480-8.

16. Siegel R, Naishadham D, Jemal A. Cancer statistics, 2012. CA Cancer J Clin. 2012;62(1):10-29.

17. Voutsina A, Tzardi M, Kalikaki A, Zafeiriou Z, Papadimitraki E, Papadakis M, et al. Combined analysis of KRAS and PIK3CA mutations, MET and PTEN expression in primary tumors and corresponding metastases in colorectal cancer. Mod Pathol. 2013;26(2):302-13.

18. Resnick MB, Routhier J, Konkin T, Sabo E, Pricolo VE. Epidermal growth factor receptor, c-MET, beta-catenin, and p53 expression as prognostic indicators in stage II colon cancer: a tissue microarray study. Clin Cancer Res. 2004;10(9):3069-75.

19. Zeng ZS, Weiser MR, Kuntz E, Chen CT, Khan SA, Forslund A, et al. c-Met gene amplification is associated with advanced stage colorectal cancer and liver metastases. Cancer Lett. 2008;265(2):258-69.

20. Liu Y, Li Q, Zhu L. Expression of the hepatocyte growth factor and c-Met in colon cancer: correlation with clinicopathological features and overall survival. Tumori. 2012;98(1):105-12.

21. Garouniatis A, Zizi-Sermpetzoglou A, Rizos S, Kostakis A, Nikiteas N, Papavassiliou AG. FAK, CD44v6, c-Met and EGFR in colorectal cancer parameters: tumour progression, metastasis, patient survival and receptor crosstalk. Int J Colorectal Dis. 2013;28(1):9-18

22. Samame Perez-Vargas JC, Biondani P, Maggi C, Gariboldi M, Gloghini A, Inno $\mathrm{A}$, et al. Role of CMET in the development and progression of colorectal cancer. Int J Mol Sci. 2013;14(9):18056-77.

23. Lau PC, Chan AT. Novel therapeutic target for head and neck squamous cell carcinoma: HGF-MET signaling pathway. Anti-cancer drugs. 2011;22(7):665-73.

24. Scagliotti GV, Novello S, von Pawel J. The emerging role of MET/HGF inhibitors in oncology. Cancer Treat Rev. 2013;39(7):793-801.

\section{Submit your next manuscript to BioMed Central and take full advantage of:}

- Convenient online submission

- Thorough peer review

- No space constraints or color figure charges

- Immediate publication on acceptance

- Inclusion in PubMed, CAS, Scopus and Google Scholar

- Research which is freely available for redistribution 